\title{
Normal Forms for Coupled Takens-Bogdanov Systems
}

David Mumo MALONZA

Department of Mathematics, Iowa State University, Ames, Iowa, 50011

E-mail:dmalo@iastate.edu

Received February 24, 2004; Accepted April 27, 2004

\begin{abstract}
The set of systems of differential equations that are in normal form with respect to a particular linear part has the structure of a module of equivariants, and is best described by giving a Stanley decomposition of that module. In this paper Groebner basis methods are used to determine a Groebner basis for the ideal of relations and a Stanley decomposition for the ring of invariants that arise in normal forms for TakensBogdanov systems. An algorithm developed by Murdock, is then used to produce a Stanley decomposition for the (normal form module) module of the equivariants from the Stanley decomposition for the ring of invariants.
\end{abstract}

\section{Introduction}

A coupled Takens-Bogdanov system has the form

$$
\left[\begin{array}{c}
\dot{x_{1}} \\
\dot{y_{1}} \\
\vdots \\
\dot{x_{n}} \\
\dot{y_{n}}
\end{array}\right]=\left[\begin{array}{lllll}
N_{2} & & & & \\
& N_{2} & & & \\
& & \ddots & & \\
& & & N_{2} & \\
& & & & N_{2}
\end{array}\right]\left[\begin{array}{c}
x_{1} \\
y_{1} \\
\vdots \\
x_{n} \\
y_{n}
\end{array}\right]+\ldots .
$$

That is,

$$
\dot{x}=N_{22 \ldots 2} x+\cdots,
$$

where $x \in \mathbb{R}^{2 n}, N_{22 \ldots 2}=\left[\begin{array}{cccc}N_{2} & & & \\ & N_{2} & & \\ & & \ddots & \\ & & & N_{2}\end{array}\right], N_{2}=\left[\begin{array}{ll}0 & 1 \\ 0 & 0\end{array}\right]$

and the dots denote higher order terms. Such systems can arise from physical problems 
in various ways. For instance, Sri Namachchivaya et al. in [14] have studied a generalized Hopf bifurcation with nonsemisimple 1:1 resonance having an equilibrium point with linear part governed by the matrix

$$
A=\left[\begin{array}{cccc}
i \omega & 1 & & \\
& i \omega & & \\
& & -i \omega & 1 \\
& & & -i \omega
\end{array}\right]
$$

The normal form for such a system contains only terms that belong to both the semisimple part of $A$ and the normal form of the nilpotent part, which is a coupled Takens-Bogdanov system with $N_{22}$. This example illustrates the physical significance of the study of normal forms for systems with nilpotent linear part. In this paper we study coupled TakensBogdanov systems with an arbitrary number of $2 \times 2$ blocks.

The problem of finding Stanley decompositions for the equivariants of $N_{22 \ldots 2}$ was first solved by Richard Cushman, Jan Sanders and Neil White [4] using a method called "covariants of special equivariants." Their method begins by creating a scalar problem that is larger than the vector problem and their procedures are derived from classical invariant theory. The method presented here is considerably simpler since it begins by studying a scalar problem (the problem of invariants) that is smaller and simpler than the vector problem (of equivariants). When the scalar problem has been solved, our approach makes it unnecessary to repeat the calculations of classical invariant theory at the level of equivariants. Instead an algorithm is given by Murdock [9] (through the construction of suffixes and their prefix rings) that converts a Stanley decomposition of the invariant ring into Stanley decomposition of the module, the normal form module.

Our method also relies on the application of methods from classical invariant theory to a scalar problem. The entire scalar algebra that we use is relevant to the problem of equivariants because it is just the ring over which the equivariants form a module. (The algebra studied in [4] is too large since it contains many polynomials having nothing to do with the equivariants.) Although our algebra does not seem to be isomorphic to a classical bracket algebra, it is very close, and we have studied it "from scratch" by Groebner basis methods rather than by borrowing classical results. It is hoped that these methods will extend to other examples, such as $N_{33 \cdots 3}$ or $N_{44 \cdots 4}$. Even though the method of covariants of special equivariants could be applied to these problems it does not lead to an algebra that is recognizable in classical terms, so one cannot immediately carry out the program of [4] for these problems. It would be necessary to study the algebra "from scratch," which would be more difficult than studying the algebra of invariants needed for our method.

Our results are based mainly on the work by Murdock in $[9,10]$, that is the application of Murdock's methods for computing Stanley decompositions for equivariants of nilpotent systems. In section one we put together some background knowledge, without proofs, for understanding the content of this paper. In section two which forms the central part of this paper, we use Groebner basis methods to compute a Groebner basis for the ideal of relations among the basic invariants and compute a Stanley decomposition for the ring of invariants. The concept of partially ordered set ring is used to develop an easy method to write down a Stanley decomposition when a Groebner basis for the ideal of relations is large. In section three we apply Murdock's algorithm to find the Stanley decomposition 
for the module of equivariants from the Stanley decomposition of the ring of invariants. The basis for normal form theory, which is well known, can be found in [3] or [10].

I thank James Murdock and Alexander Burstein for their valuable suggestions and comments.

\section{Invariants and Equivariants}

Let $\mathcal{P}_{j}\left(\mathbb{R}^{n}, \mathbb{R}^{m}\right)$ denote the vector space of homogeneous polynomials of degree $j$ on $\mathbb{R}^{n}$ with coefficients in $\mathbb{R}^{m}$, where $\mathbb{R}$ denotes the set of real numbers. Let $\mathcal{P}\left(\mathbb{R}^{n}, \mathbb{R}^{m}\right)$ be the vector space of all such polynomials of any degree and let $\mathcal{P}_{*}\left(\mathbb{R}^{n}, \mathbb{R}^{m}\right)$ be the vector space of formal power series. If $m=1, \mathcal{P}_{*}\left(\mathbb{R}^{n}, \mathbb{R}\right)$ becomes a ring of (scalar) formal power series on $\mathbb{R}^{n}$. If $f \in \mathcal{P}_{i}\left(\mathbb{R}^{n}, \mathbb{R}\right)$ and $v \in \mathcal{P}_{j}\left(\mathbb{R}^{n}, \mathbb{R}^{m}\right)$, then $f v \in \mathcal{P}_{i+j}\left(\mathbb{R}^{n}, \mathbb{R}^{m}\right)$, making $\mathcal{P}_{*}\left(\mathbb{R}^{n}, \mathbb{R}^{m}\right)$ a module over $\mathcal{P}_{*}\left(\mathbb{R}^{n}, \mathbb{R}\right)$. In the same way $\mathcal{P}\left(\mathbb{R}^{n}, \mathbb{R}^{m}\right)$ is a module over $\mathcal{P}\left(\mathbb{R}^{n}, \mathbb{R}\right)$. From the viewpoint of smooth vector fields it is most natural to work with formal power series, but since in practice these must be truncated at some degree, it is sufficient to work with polynomials.

For any nilpotent matrix, N, define the Lie operator

$$
L_{N}: \mathcal{P}_{j}\left(\mathbb{R}^{n}, \mathbb{R}^{n}\right) \rightarrow \mathcal{P}_{j}\left(\mathbb{R}^{n}, \mathbb{R}^{n}\right)
$$

by

$$
\left(L_{N} v\right) x=v^{\prime}(x) N x-N v(x)
$$

and define the differential operator

$$
\mathcal{D}_{N x}: \mathcal{P}_{j}\left(\mathbb{R}^{n}, \mathbb{R}\right) \rightarrow \mathcal{P}_{j}\left(\mathbb{R}^{n}, \mathbb{R}\right)
$$

by

$$
\left(\mathcal{D}_{N x} f\right)(x)=f^{\prime}(x) N x=(N x \cdot \nabla) f(x) .
$$

Then $\mathcal{D}_{N}$ is a derivation of the ring $\mathcal{P}\left(\mathbb{R}^{n}, \mathbb{R}\right)$, meaning that

$$
\mathcal{D}_{N}(f g)=\left(\mathcal{D}_{N} f\right) g+f\left(\mathcal{D}_{N} g\right) \text {. }
$$

In addition

$$
L_{N}(f v)=\left(\mathcal{D}_{N} f\right) v+f L_{N} v .
$$

Recall that, if $v$ is a vector field and $f$ is a scalar field, then $\mathcal{D}_{v(x)} f$ is a scalar field called the derivation of $f$ along (the flow of) $v(x)$. We write $\mathcal{D}_{N}$ for $\mathcal{D}_{N x}$ to denote the derivation along the linear vector field $N x$.

A function $f$ is called an invariant of (the flow of) $N x$ if $\left.\frac{\partial}{\partial t} f\left(e^{N t} x\right)\right|_{t=0}=0$ or equivalently $f \in \operatorname{ker} \mathcal{D}_{N}$. Since

$$
\begin{aligned}
\mathcal{D}_{N}(f+g) & =\mathcal{D}_{N} f+\mathcal{D}_{N} g \\
\mathcal{D}_{N}(f g) & =f \mathcal{D}_{N} g+g \mathcal{D}_{N} f,
\end{aligned}
$$


it follows that, if $f$ and $g$ are invariants, then so are $f+g$ and $f g$; that is, ker $\mathcal{D}_{N}$ is both a vector space over $\mathbb{R}$ and also a subring of $\mathcal{P}\left(\mathbb{R}^{n}, \mathbb{R}\right)$, known as the ring of invariants. Similarly a vector field $v$ is called an equivariant of (the flow of) $N x$ if $\left.\frac{\partial}{\partial t}\left(e^{-N t} v\left(e^{N t} x\right)\right)\right|_{t=0}=0$, that is, $v \in \operatorname{ker} L_{N}$.

A vector space $\mathcal{N} \subset \mathcal{P}\left(\mathbb{R}^{n}, \mathbb{R}^{n}\right)$ is called a normal form style if

$$
\mathcal{P}\left(\mathbb{R}^{n}, \mathbb{R}^{n}\right)=\operatorname{im} L_{N} \oplus \mathcal{N}
$$

There are two normal form styles in common use for nilpotent systems, the inner product normal form and the sl(2) normal form. The inner product normal form is defined by $\mathcal{P}\left(\mathbb{R}^{n}, \mathbb{R}^{n}\right)=\operatorname{im} L_{N} \oplus \operatorname{ker} L_{N^{*}}$, where $N^{*}$ is the conjugate transpose of the nilpotent matrix $N$. It follows from (2.4), applied to $N^{*}$, that ker $L_{N^{*}}$ is a module over ker $\mathcal{D}_{N^{*}}$. This is the inner product normal form module.

To define the $s l(2)$ normal form one firstly sets $\mathrm{X}=\mathrm{N}$ and constructs matrices $\mathrm{Y}$ and Z such that

$$
[X, Y]=Z, \quad[Z, X]=2 X, \quad[Z, Y]=-2 Y .
$$

An example of such an $\operatorname{sl}(2) \operatorname{triad}\{X, Y, Z\}$ is

$$
X=\left(\begin{array}{llll}
0 & 1 & 0 & 0 \\
0 & 0 & 0 & 0 \\
0 & 0 & 0 & 1 \\
0 & 0 & 0 & 0
\end{array}\right), \quad Y=\left(\begin{array}{llll}
0 & 0 & 0 & 0 \\
1 & 0 & 0 & 0 \\
0 & 0 & 0 & 0 \\
0 & 0 & 1 & 0
\end{array}\right), \quad Z=\left(\begin{array}{cccc}
1 & 0 & 0 & 0 \\
0 & -1 & 0 & 0 \\
0 & 0 & 1 & 0 \\
0 & 0 & 0 & -1
\end{array}\right)
$$

Having obtained the triad $\{X, Y, Z\}$ one can create two additional triads $\{X, y, Z\}$ and $\{\mathrm{X}, \mathrm{Y}, \mathrm{Z}\}$ as follows:

$$
x=\mathcal{D}_{Y}, \quad y=\mathcal{D}_{X}, \quad z=\mathcal{D}_{Z}
$$

and

$$
\mathrm{X}=L_{\mathrm{Y}}, \quad \mathrm{Y}=L_{\mathrm{X}}, \quad \mathrm{Z}=L_{\mathrm{Z}}
$$

The first of these is a triad of differential operators and the second is a triad of Lie operators. Both the operators $\{X, Y, Z\}$ and $\{X, Y, Z\}$ inherit the triad properties (2.8); that is,

$$
[x, y]=z, \quad[z, x]=2 x, \quad[z, y]=-2 y
$$

and

$$
[\mathrm{X}, \mathrm{Y}]=\mathrm{Z}, \quad[\mathrm{Z}, \mathrm{X}]=2 \mathrm{X}, \quad[\mathrm{Z}, \mathrm{Y}]=-2 \mathrm{Y} .
$$

The name $s l(2)$ normal form style results from the fact that $\{X, Y, Z\}$ span a Lie algebra of $n \times n$ matrices isomorphic to the Lie algebra $\operatorname{sl}(2)$. Observe that the operators $\{\mathrm{X}, \mathrm{Y}, \mathrm{Z}\}$ 
map each $\mathcal{P}\left(\mathbb{R}^{n}, \mathbb{R}^{n}\right)$ into itself. It then follows from Theorem 1 and the representation theory of $\operatorname{sl}(2)$ in [5] that

$$
\mathcal{P}\left(\mathbb{R}^{n}, \mathbb{R}^{n}\right)=\operatorname{im} \mathrm{Y} \oplus \operatorname{ker} \mathrm{X}=\operatorname{im} \mathrm{X} \oplus \operatorname{ker} \mathrm{Y} .
$$

Clearly the ker $X$ is a subring of $\mathcal{P}\left(\mathbb{R}^{n}, \mathbb{R}\right)$, the ring of invariants and it follows from (2.4) that ker $\mathrm{X}$ is a module over this subring. This is the $s l(2)$ normal form module. In this paper we are concerned only with the $\operatorname{sl}(2)$ normal forms.

Let $N: V \rightarrow V$ be nilpotent. A lower Jordan chain for $N$ of length $\ell$ is an ordered sequence of $\ell$ nonzero vectors of the form $\left\{v, N v, N^{2} v, \ldots, N^{\ell-1} v\right\}$ with $N^{\ell} v=0$ and $v \notin \operatorname{im} N$. The vector $v$ is called the chain top,$N^{\ell-1} v$ the chain bottom and the height of the chain top equals the chain length $\ell$. The notions of top, bottom and height are represented by listing the vectors from top to bottom with the map $N$ pointing downwards.

Given a vector space $\mathrm{V}$ and a triad $\{\mathrm{X}, \mathrm{Y}, \mathrm{Z}\}$, there exist two splittings that provide complements to the images of $\mathrm{X}$ and $\mathrm{Y}$, namely

$$
\mathrm{V}=\operatorname{im} X \oplus \operatorname{ker} \mathrm{Y}=\operatorname{im} Y \oplus \operatorname{ker} X .
$$

The existence of these splittings is established in the following theorem which is Theorem 2.5.2 in [10].

Theorem 1. Suppose that $V$ is a finite dimensional vector space and $\{X, Y, Z\}$ is an $\operatorname{sl}(2)$ triad of linear operators on $V$. Then:

1. $X$ and $Y$ are nilpotent.

2. $Z$ is diagonalizable and has integer eigenvalues (called weights).

3. $\operatorname{ker} X$ has basis consisting of weight vectors(eigenvectors of $Z$ ).

4. Any basis $\left\{v_{1}, v_{2}, \ldots, v_{s}\right\}$ of $\operatorname{ker} X$ consisting of weight vectors can be taken as a set of chain tops for lower Jordan chains for $Y$; that is, each sequence $v_{j}, Y v_{j}, Y^{2} v_{j}, \ldots$ terminates with 0 and constitutes an (independent) lower Jordan chain for $Y$ so that the nonzero vectors of the form $Y^{i} v_{j}$ form a basis for $V$. In particular it follows that

$$
V=\operatorname{ker} X \oplus \operatorname{im} Y \text {. }
$$

5. The vectors $Y^{i} v_{j}$ are also weight vectors with weights given by

$$
\operatorname{wt}\left(Y^{i} v_{j}\right)=\operatorname{wt}\left(v_{j}\right)-2 i .
$$

6. The length of the chain headed by $v_{j}$ is $\mathrm{wt}\left(v_{j}\right)+1$.

7. The action of $X$ on the basis vectors is given by

$$
X\left(Y^{i} v_{j}\right)=\operatorname{pr}\left(Y^{i} v_{j}\right)\left(Y^{i-1} v_{j}\right)
$$


where $\operatorname{pr}\left(Y^{i} v_{j}\right)$ is the nonzero constant $\operatorname{pr}\left(Y^{i} v_{j}\right)=\operatorname{wt}\left(v_{j}\right)+\operatorname{wt}\left(Y v_{j}\right)+\cdots+\operatorname{wt}\left(Y^{i-1} v_{j}\right)$. The constant $\operatorname{pr}\left(Y^{i} v_{j}\right)$ is called the pressure on $Y^{i} v_{j}$ because it is the sum of the weights of the vectors above $Y^{i} v_{j}$ in its Jordan chain.

8. The number of chain tops of weight $w \geq 0$ equals $m(w)-m(w+2)$, where $m(w)$ is the multiplicity of $w$ as an eigenvalue of $Z$.

This theorem may be used in the converse manner to construct $s l(2)$ triads such as (2.9). That is, given the nilpotent matrix $\mathrm{X}$ in upper Jordan form, $\mathrm{Y}$ must contain the pressures and $\mathrm{Z}$ the weights. For each Jordan block of $\mathrm{X}$ the weights form a string of integers differing by 2 and symmetrically arranged around zero. The pressures can then be found by taking partial sums of the weights. Our major application of Theorem 1 is to calculate ker $\mathcal{X}$, the ring of invariants.

Let $k\left[x_{1}, \cdots, x_{n}\right]$ be a polynomial ring over the field $k$, let $f_{i} \in k\left[x_{1}, \cdots, x_{n}\right], 1 \leq i \leq m$ and let $\varphi: k\left[y_{1}, \cdots, y_{m}\right] \rightarrow k\left[x_{1}, \cdots, x_{n}\right]$ be the ring homomorphism defined by

$$
\varphi: y_{i} \mapsto f_{i}
$$

Let $h \in k\left[y_{1}, \cdots, y_{m}\right]$ so that $h\left(y_{1}, \cdots, y_{m}\right)=\sum_{\mu} C_{\mu} y_{1}^{\mu_{1}} \cdots y_{m}^{\mu_{m}}$ with $C_{\mu} \in k, \mu=$ $\left(\mu_{1}, \cdots, \mu_{m}\right) \in \mathbb{N}^{m}$, and only finitely many $C_{\mu} s$ nonzero. Then

$$
\varphi(h)=h\left(f_{1}, \cdots, f_{m}\right) \in k\left[x_{1}, \cdots, x_{n}\right] .
$$

The kernel of $\varphi$,

$$
\operatorname{ker} \varphi=\left\{h \in k\left[y_{1}, \cdots, y_{m}\right]: \varphi(h)=0\right\}
$$

is called the ideal of relations among the polynomials $f_{1}, \cdots, f_{m}$. This ideal plays an important role in this paper.

The following theorem, the proof of which can be found in [1], provides an algorithm for computing the kernel of $\varphi$ or more precisely a Groebner basis for the kernel of $\varphi$.

Theorem 2. Let $K=\left\langle y_{1}-f_{1}, \cdots, y_{m}-f_{m}\right\rangle \subseteq k\left[y_{1}, \cdots, y_{m}, x_{1}, \cdots, x_{n}\right]$. Then $\operatorname{ker} \varphi=$ $K \cap k\left[y_{1}, \cdots, y_{m}\right]$.

Definition 1. Let $\mathcal{R} \subset \mathbb{R}\left[x_{1}, \cdots, x_{n}\right]$ be a subring of the ring of polynomials. Let $\mathcal{R}_{1}, \cdots, \mathcal{R}_{s}$ be subrings of $\mathcal{R}$ and let $f_{1}, \cdots, f_{s} \in \mathbb{R}\left[x_{1}, \cdots, x_{n}\right]$. If

$$
\mathcal{R}=\mathcal{R}_{1} f_{1} \oplus \mathcal{R}_{2} f_{2} \oplus \cdots \oplus \mathcal{R}_{s} f_{s}
$$

then (2.15) is called a Stanley decomposition of $\mathcal{R}$ and every element of $\mathcal{R}$ can be written uniquely as $\sum_{i=1}^{s} g_{i} f_{i}$ for $g_{i} \in \mathcal{R}_{i}, i=1, \cdots, s$.

Next we describe a procedure for obtaining a Stanley decomposition for ker $\mathbf{X}$ given a Stanley decomposition for ker $\mathcal{X}$, where $\mathcal{X}$ and $X$ are defined as in equations (2.10) and (2.11) respectively. 
Let $N_{r_{1}, r_{2}, \ldots, r_{k}}$ be an $n \times n$ block diagonal nilpotent matrix with upper Jordan blocks of sizes $r_{1}, r_{2}, \ldots, r_{k}$ with $r_{1}+r_{2}+\ldots+r_{k}=n$. Let $R_{i}=r_{1}+r_{2}+\cdots r_{i}, i=1,2, \ldots, k$, so that $R_{1}, R_{2}, \cdots, R_{k}$ are the row numbers of the bottom rows of the Jordan blocks.

Define a map

$$
\widetilde{\varphi}: \mathcal{P}\left(\mathbb{R}^{n}, \mathbb{R}^{n}\right) \longrightarrow \mathcal{P}\left(\mathbb{R}^{n}, \mathbb{R}^{k}\right)
$$

by

$$
\widetilde{\varphi}\left(v_{1}, \ldots, v_{n}\right)=\left(v_{R_{1}}, \ldots, v_{R_{k}}\right)
$$

Clearly $\widetilde{\varphi}$ is a homomorphism of modules over $\mathcal{P}\left(\mathbb{R}^{n}, \mathbb{R}\right)$. Let $\varphi$ be the restriction of $\widetilde{\varphi}$ to ker $\mathrm{X}$. Hence we have the following theorem, which is Theorem 1 in [9].

Theorem 3. The image of $\varphi$ is $\operatorname{ker} X^{r_{1}} \oplus \operatorname{ker} X^{r_{2}} \oplus \ldots \oplus \operatorname{ker} X^{r_{k}}$ and the mapping $\varphi: \operatorname{ker} \mathrm{X} \longmapsto \operatorname{ker} X^{r_{1}} \oplus \operatorname{ker} X^{r_{2}} \oplus \ldots \oplus \operatorname{ker} \mathcal{X}^{r_{k}}$ is an isomorphism of modules over the ring ker $X$.

Proof. Observe that, if $f \in \operatorname{ker} X$ and $g \in \mathcal{P}\left(\mathbb{R}^{n}, \mathbb{R}\right)$, then $\mathcal{X}(f g)=f X g$. It follows that, if $g \in \operatorname{ker} X^{r}$ (for any r), then $f g \in \operatorname{ker} \mathbf{X}^{r}$ : that is, ker $X^{r}$ is a module over ker $X$. The rest of the proof becomes clear after we consider the example

$$
\mathbf{N}_{222}=\left[\begin{array}{cccccc}
0 & 1 & & & & \\
0 & 0 & & & & \\
& & 0 & 1 & & \\
& & 0 & 0 & & \\
& & & & 0 & 1 \\
& & & & 0 & 0
\end{array}\right]
$$

In this case it follows that $\widetilde{\varphi}\left(v_{1} \cdots, v_{6}\right)=\left(v_{2}, v_{4}, v_{6}\right)$ and, if $v \in$ ker $\mathcal{X}$, then $\mathcal{X} v_{1}=$ $0, X_{v_{2}}=v_{1}, X_{v_{3}}=0, X_{v_{4}}=v_{3}, X_{v_{5}}=0, X_{v_{6}}=v_{5}$. These conditions imply that $X^{2} v_{2}=0, X^{2} v_{4}=0$ and $X^{2} v_{6}=0$ so that $\varphi(v)=\left(v_{2}, v_{4}, v_{6}\right) \in \operatorname{ker} X^{2} \oplus \operatorname{ker} X^{2} \oplus$ ker $X^{2}$ and also show that $v \in \operatorname{ker} X$ can be reconstructed from $\left(v_{2}, v_{4}, v_{6}\right)$ by the reconstruction map

$$
\varphi^{-1}\left(v_{2}, v_{4}, v_{6}\right)=\left[\begin{array}{c}
x v_{2} \\
v_{2} \\
x v_{4} \\
v_{4} \\
x v_{6} \\
v_{6}
\end{array}\right]
$$

Thus $\widetilde{\varphi}$ is invertible. Since it is a module homomorphism, it is an isomorphism.

The following Lemma, found in [10] on page 243, also plays a crucial role in determining Stanley decompositions. 
Lemma 1. If $h \in \mathcal{P}_{j}\left(\mathbb{R}^{n}, \mathbb{R}\right)$ belong to the $\operatorname{ker} \mathcal{D}_{N}^{r_{s}}$ and $e_{i}, i=1, \cdots, n$ is the standard basis for $\mathbb{R}^{n}$, then the vector polynomial $v_{\{s, h\}}$ defined by

$$
v_{\{s, h\}}=\sum_{i=0}^{r_{s}-1}\left(\mathcal{D}_{N}^{i} h\right) e_{R_{s}-i}
$$

belongs to ker $\mathrm{L}_{N}$.

Definition 2. Let $J$ be a monomial ideal, the monomials belonging to $J$ are called nonstandard monomials. The standard monomials with respect to this ideal are the monomials that do not belong to it.

The following lemma, which is Lemma 1 in [9], forms the basis for obtaining the Stanley decomposition of ker $\mathbf{X}$.

Lemma 2. Let $\mathcal{R}$ be any subring of ker $\mathcal{X}$ generated by homogeneous polynomials $I_{1}, \cdots, I_{s}$ in $x=\left(x_{1}, \cdots, x_{n}\right)$ that are weight vectors for the triad $\{x, y, Z\}$ and let $\mathcal{R}_{i k}$ be the vector subspace of $\mathcal{R}$ consisting of polynomials homogeneous in $x$ of degree $i$ and weight $k$. Let $a$ Groebner basis for the relations of $I_{1}, \cdots, I_{s}$ be selected. Then:

1. The standard monomials in $I_{1}, \cdots, I_{s}$ (with respect to the given Groebner basis) having degree $i$ (in $x$ ) and weight $k$ form a basis for $\mathcal{R}_{i k}$.

2. If $\mathcal{R}=\operatorname{ker} \mathcal{X}$, the standard monomials of degree $i$ form a set of chain tops for the chains in $\mathcal{P}\left(\mathbb{R}^{n}, \mathbb{R}\right)$.

According to this Lemma the chain tops of $\mathcal{P}\left(\mathbb{R}^{n}, \mathbb{R}\right)$ under the triad $\{X, y, z\}$ may be taken to be the standard monomials in the basic invariants $I_{1}, \cdots, I_{s}$ with respect to the given Stanley decomposition of ker $X$. The chains under the chain tops can be obtained by repeated application of $y$. A vector space basis for ker $X^{r}$ can be obtained by computing the iterates down to depth $r$.

Let $f$ be a standard monomial of degree $j$ (in $\mathrm{x}$ ) and let $y^{i} f$ be a nonzero entry in the chain under $f$. We define $g \in \mathcal{P}_{i}\left(\mathbb{R}^{n}, \mathbb{R}\right)$ to be a replacement for $y^{i} f$ if $X^{i} g$ is a nonzero multiple of $f$. Hence we have the following lemma found in [9] on page 219 and also in [10] on page 253 .

Lemma 3. Let $f$ be a standard monomial. A replacement for $y^{r} f$ can be found by placing $r$ copies of $y$ arbitrarily in front of the various factors of $f$ as long as the result is not zero.

Note that the maximum power of $y$ that can be applied to an invariant equals the weight (length - 1) of the invariant. By Lemma 3 above, we think of each standard monomial as being written without powers, so that $I_{2}^{3} I_{3}^{2}$ appears as $I_{2} I_{2} I_{2} I_{3} I_{3}$. Apply $y$ repeatedly to the last factor until the power of $y$ equals its weight, then to the factor before that and so on, stopping when the total number of factors of $y$ reaches $r-1$ (for the construction of replacements for the chain elements under a standard monomial to depth $r$ ). Each replacement constructed in this manner contains two parts, a prefix, which is itself a standard monomial and contains no $y$, and a suffix, which begins with the first occurrence of $y$. It is clear that no basic invariant $I$ of weight zero can appear in a suffix since $y I=0$. We call a such basic invariant trivial. 
The next step is to describe the set of prefixes that can occur with any given suffix. Let $S$ be a suffix and let $g$ be the standard monomial that results from deleting all occurrences of $y$ in $S$. We call $g$ a stripped suffix. Let $f$ be any other standard monomial. Then $f S$ occurs as a replacement, that is, $f$ is a prefix for $S$, precisely when the following two conditions are satisfied:

1. $f g$ is a standard monomial (so that $f g$ occurs as a chain top);

2. The factors $f g$ are correctly ordered, equivalently, the final factor of $f$ either precedes or equals the first factor of $g$.

Let $m_{1}, \cdots, m_{p}$ be the leading monomials of the Groebner basis $\gamma_{1}, \cdots, \gamma_{p}$ for the basic invariants $I_{1}, \cdots, I_{t}$. Given $g$ the condition (1) for $f g$ to be standard is that $f$ be not divisible by any of the monomials $m_{i}^{\prime}=m_{i} / \operatorname{gcd}\left(m_{i}, g\right)$. Let the first basic invariant appearing in $g$ (from the left) be $I_{i(g)}$. Then the condition (2) for $f g$ to be correctly ordered is that $f$ be not divisible by $I_{i(g)-1}, \cdots, I_{t}$ (ordering the basic invariants by $I_{i}<I_{j}$ if $j<i$ ). Therefore the prefix monomials $f$ associated with the given stripped suffix $g$ are the standard monomials with respect to the (new) ideal $J_{S}=\left\langle m_{1}^{\prime}, \cdots, m_{p}^{\prime}, I_{i(g)-1}, \cdots, I_{t}\right\rangle$. Now let $f$ be a prefix monomial associated with a given suffix $S$. Then the collection of polynomials which are linear combination of such prefix monomials for a given suffix $S$ is a ring, called the prefix ring for $S$, which has a Stanley decomposition (defined by its standard prefix monomials). This Stanley decomposition denoted by $P(S)$ is called the Stanley decomposition of the prefix ring for the suffix $S$. We conclude this section with the following theorem which is Theorem 3 in [9] and is useful for computing ker $X^{r}$.

Theorem 4. A Stanley decomposition for ker $X^{r}$ is given by

$$
\operatorname{ker} X^{r}=S D(\operatorname{ker} X) \oplus\left(\bigoplus_{S} P(S) S\right)
$$

or, equivalently by the above discussion,

$$
\operatorname{ker} X^{r}=S D(\operatorname{ker} x) \oplus\left(\bigoplus_{S}\left(\operatorname{ker} x / J_{S}\right) S\right)
$$

where $S D(\operatorname{ker} X)$ is the Stanley decomposition of ker $X$ and $P(S)$ is the Stanley decomposition of the prefix ring corresponding to suffix $S$.

\section{The Ring of Invariants}

In this section we find a Groebner basis and a Stanley decomposition for $\mathcal{R}=$ ker $\mathcal{X}$, the ring of invariants, for coupled Takens-Bogdanov systems;

$$
\dot{x}=N_{22 \ldots 2} x+\cdots \text {. }
$$

According to Murdock [10] four steps are required to complete this calculation.

- Compute a finite set of invariants $I_{1}, I_{2}, \cdots, I_{s}$ for ker $X$ called basic invariants which suffices to generate all invariants up to some chosen degree $j$. 
- Compute a Groebner basis for the ideal of relations among the basic invariants.

- From the Groebner basis determine a Stanley decomposition for the ring $\mathcal{R}$ of polynomials in the basic invariants.

- From the Stanley decomposition set up a two-variable generating function called the table function (Hilbert function, see [10], chapter 4) and use it to test that $\mathcal{R}$ is in fact all of ker $X$. If it is not, then not all of the basic invariants have been found. In that case return back to the first step and increase the value of $j$.

In step one the basic invariants can be found by methods outlined in [10] and are given by the following lemma.

Lemma 4. Let $\alpha_{i}=x_{i}$ for $1 \leq i \leq n$ and let $\beta_{i j}=x_{i} y_{j}-x_{j} y_{i}$ for $1 \leq i<j \leq n$. Then $\alpha_{i}$ and $\beta_{i j}$ are invariants, that is, elements of ker $\mathcal{X}$.

Proof. Since $X=x_{1} \frac{\partial}{\partial y_{1}}+x_{2} \frac{\partial}{\partial y_{2}}+\cdots+x_{n} \frac{\partial}{\partial y_{n}}$ and $\gamma$ is an element of ker $X$ if $\mathcal{X}(\gamma)=0$, it follows immediately that $\alpha_{i}$ and $\beta_{i j}$ are invariants of $\mathcal{X}$.

In step two to compute a Groebner basis for the ideal of relations among the basic invariants we proceed as follows:

- Introduce slack variables, $\left\{z_{i} ; 1 \leq i \leq n\right\}$ and $\left\{w_{i j} ; 1 \leq i<j \leq n\right\}$, and let $\{x, y\}=\left\{x_{1}, y_{1}, x_{2}, y_{2}, \cdots, x_{n}, y_{n}\right\}$ and $\{z, w\}=\left\{z_{i} ; 1 \leq i \leq n, w_{i j} ; 1 \leq i<j \leq n\right\}$.

- Let $\Phi: \mathbb{R}[z, w] \rightarrow \mathbb{R}[x, y]$ be a map defined by

$$
\begin{aligned}
z_{i} & \mapsto \alpha_{i} \\
w_{i j} & \mapsto \beta_{i j} .
\end{aligned}
$$

Definition 3. A relation among the basic invariants is a function $f(z, w)$ such that $f(\alpha, \beta)=0$, that is, $f \in \operatorname{ker} \Phi$, the ideal of relations among the polynomial invariants.

We apply Groebner basis methods to find a Groebner basis for the kernel of $\Phi$; see [1] and [2] for more details. Let

$$
\begin{aligned}
g_{i} & =\alpha_{i}-z_{i}, \quad 1 \leq i \leq n \\
h_{i j} & =\beta_{i j}-w_{i j}, \quad 1 \leq i<j \leq n
\end{aligned}
$$

and set $F=\left\{g_{i}, h_{i j}\right\}$. By Theorem 2 we first compute a Groebner basis $\mathbf{G}$ for the polynomial ideal $\mathrm{K}=\left\langle\alpha_{i}-z_{i}, \beta_{i j}-w_{i j}\right\rangle$ in $\mathbb{R}[x, y, z, w]$ generated by $F$. The polynomials in $\mathbf{G}$ without $x$ and $y$ variables form a Groebner basis $\mathbf{H}$ for the kernel of $\Phi$. We now state one of the main theorems of the Groebner basis method found in [1], [2] and [6].

Definition 4. Fix a monomial order and let $G=\left\{g_{1}, \cdots, g_{t}\right\} \subset k\left[x_{1}, \cdots, x_{n}\right]$. Given $f \in k\left[x_{1}, \cdots, x_{n}\right]$ we say that $f$ reduces to zero modulo $G$, written $f \longrightarrow_{G} 0$, if $f$ can be written in the form $f=a_{1} g_{1}+\cdots, a_{t} g_{t}, a_{i} \in k\left[x_{1}, \cdots, x_{n}\right]$. As usual $S(f, g)$ denotes the S-polynomial of $f$ and $g$. 
Theorem 5. (Buchberger's theorem) $A$ basis $G=\left\{g_{1}, \cdots, g_{t}\right\}$ for an ideal $I$ is a Groebner basis if and only if $S\left(g_{i}, g_{j}\right) \longrightarrow_{G} 0$ for all $i \neq j$, that is, if and only if for all pairs $i \neq j$ the remainder on division of $S\left(g_{i}, g_{j}\right)$ by $G$ is zero.

Corollary 6. (Buchberger's First Criterion) Given a finite set $G \subset k\left[x_{1}, \cdots, x_{n}\right]$, suppose that we have $f, g \in G$ such that $\operatorname{gcd}(\operatorname{lm}(f), \operatorname{lm}(g))=1$. Then $S(f, g) \longrightarrow_{G} 0$.

With this criterion certain S-polynomials are guaranteed to reduce to zero without doing any calculations.

The following theorem, which is Theorem 2.2.3 in [8], provides the required bases $\mathbf{G}$ and $\mathbf{H}$ and forms the basis for the results in this paper.

Theorem 7. The set $\mathbf{G}= \begin{cases}g_{i}=x_{i}-z_{i}, & 1 \leq i \leq n ; \\ h_{i j}=x_{i} y_{j}-x_{j} y_{i}-w_{i j}, & 1 \leq i<j \leq n ; \\ r_{i j}=y_{i} z_{i}-y_{j} z_{i}+w_{i j}, & 1 \leq i<j \leq n ; \\ r_{i j k}=y_{i} w_{j k}-y_{j} w_{i k}+y_{k} w_{i j}, & 1 \leq i<j<k \leq n ; \\ r_{i j k}^{\prime}=z_{i} w_{j k}-z_{j} w_{i k}+w_{i j} z_{k}, & 1 \leq i<j<k \leq n ; \\ r_{i j k l}=w_{i l} w_{j k}-w_{i k} w_{j l}+w_{i j} w_{k l}, & 1 \leq i<j<k<\leq n,\end{cases}$

is a Groebner basis for the polynomial ideal $K=\langle F\rangle$.

The proof of this theorem is accomplished by the following 14 lemmas, the proofs of which are based on checking that all S-polynomials reduce to zero upon division by $G$ are found in chapter 2 of [8]. For convenience we provide proofs to the first five lemmas.

Lemma 5. $S\left(g_{i}, g_{j}\right) \longrightarrow \mathbf{G} 0$ for $i \neq j$.

Proof. This follows immediately from Buchberger's first criterion, Corollary 6 .

Lemma 6. a. $S\left(g_{i}, h_{j k}\right) \longrightarrow_{\mathbf{G}} 0$ for $i \neq j$

b. $S\left(g_{i}, h_{j k}\right) \longrightarrow \mathbf{G} 0$ for $i=j$.

Proof. a. Follows from Buchberger's first criterion, Corollary 6.

b. Let $i=j$. Since $S\left(g_{j}, h_{j k}\right)=x_{k} y_{j}-y_{k} z_{j}+w_{j k}=y_{i} g_{k}+r_{j k}, S\left(g_{j}, h_{j k}\right) \longrightarrow \mathbf{G} 0$.

Lemma 7. a. $S\left(h_{i j}, h_{k l}\right) \longrightarrow \mathbf{G} 0$ for $i \neq k, j \neq l$

b. $S\left(h_{i j}, h_{k l}\right) \longrightarrow \mathbf{G} 0$ for $i=k, j \neq l$

c. $S\left(h_{i j}, h_{k l}\right) \longrightarrow \mathbf{G} 0$ for $i \neq k, j=l$.

Proof. a. Follows from Buchberger's first criterion, Corollary 6.

b. Suppose $k=i$. Then $i<j<l$ and $S\left(h_{i j}, h_{i l}\right)=-x_{j} y_{i} y_{l}+x_{k} y_{i} y_{j}-y_{l} w_{i j}+y_{j} w_{i l}=$ $-y_{i} h_{j l}+r_{i j l}$. Hence $S\left(h_{i j}, h_{i l}\right) \longrightarrow \mathbf{G} 0$. 
c. Suppose $l=j$. There are two analogous cases to consider, $i<k<j$ and $k<i<$ $j$. Suppose $i<k<j$. Since $S\left(h_{i j}, h_{k j}\right)=x_{i} x_{j} y_{k}-x_{k} x_{j} y_{i}+x_{i} w_{j k}-x_{k} w_{i j}=$ $x_{j} h_{i k}-w_{i k} g_{j}+w_{k j} g_{i}-w_{i j} g_{k}+r_{i k j}$. The other case is treated similarly. Hence $S\left(h_{i j}, h_{k j}\right) \longrightarrow \mathbf{G} 0$.

Lemma 8. a. $S\left(h_{i j}, r_{k l}\right) \longrightarrow \mathbf{G} 0$ for $j \neq k$

b. $S\left(h_{i j}, r_{k l}\right) \longrightarrow \mathbf{G} 0$ for $k=j$.

Proof. a. Follows from Buchberger's first criterion, Corollary 6.

b. Suppose $k=j$. Then $i<j<l$ and $S\left(h_{i j}, r_{j l}\right)=x_{i} y_{l} z_{j}-x_{j} y_{i} z_{l}-x_{i} w_{j l}-z_{l} w_{i j}=$ $z_{j} h_{i l}-x_{j} r_{i l}+x_{l} r_{i j}-z_{i} h_{j l}-w_{j l} g_{i}+w_{i l} g_{j}-w_{i j} g_{l}-2 r_{i j l}$. Hence $S\left(h_{i j}, r_{j l}\right) \longrightarrow \mathbf{G} 0$.

Lemma 9. a) $S\left(h_{i j}, r_{k l s}\right) \longrightarrow \mathbf{G} 0$ for $j \neq k$

b) $S\left(h_{i j}, r_{k l s}\right) \longrightarrow \mathbf{G} 0$ for $k=j$

Proof. a) Follows from Buchberger's first criterion, Corollary 6.

b) Suppose $k=j$. Since $i<j<l<s$ and $S\left(h_{i j}, r_{j l s}\right)=x_{i} y_{l} w_{j s}-x_{i} y_{s} w_{j l}-x_{j} y_{i} w_{l s}-$ $w_{i j} w_{l s}=w_{j s} h_{i l}-w_{j l} h_{i s}-x_{j} r_{i l s}+x_{l} r_{i j s}-x_{s} r_{i j l}-w_{i s} h_{j l}+w_{i l} h_{j s}-w_{i j} h_{l s}+2 r_{i j l s}$, $S\left(h_{i j}, r_{j l s}\right) \longrightarrow \mathbf{G} 0$.

The rest of the proofs follow similarly.

Lemma 10. a. $S\left(r_{i j}, r_{s t}\right) \longrightarrow_{\mathbf{G}} 0$ for $s \neq i$ and $t \neq j$

b. $S\left(r_{i j}, r_{s t}\right) \longrightarrow \mathbf{G} 0$ for $s=i$, and $t \neq j$

c. $S\left(r_{i j}, r_{s t}\right) \longrightarrow \mathbf{G} 0$ for $r \neq i$, and $t=j$.

Lemma 11. a) $S\left(r_{i j}, r_{k l s}\right) \longrightarrow \mathbf{G} 0$ for $i \neq k$

b) $S\left(r_{i j}, r_{k l s}\right) \longrightarrow \mathbf{G} 0$ for $k=i$.

Lemma 12. a) $S\left(r_{i j}, r_{l s t}^{\prime}\right) \longrightarrow_{\mathbf{G}} 0$ for $l \neq j$

b) $S\left(r_{i j}, r_{k l s}\right) \longrightarrow \mathbf{G} 0$ for $r=j$.

Lemma 13. a. $S\left(r_{i j k}, r_{l s t}^{\prime}\right) \longrightarrow_{\mathbf{G}} 0$ for $l \neq i$ and $s \neq j$ or $k \neq t$

b) $S\left(r_{i j k}, r_{l s t}^{\prime}\right) \longrightarrow \mathbf{G} 0$ for $l=i$ and $t \neq j$

c). $S\left(r_{i j k}, r_{l s t}^{\prime}\right) \longrightarrow \mathbf{G} 0$ for $l \neq i, s=j$ and $t=k$.

Lemma 14. a. $S\left(r_{i j k}, r_{l s t}^{\prime}\right) \longrightarrow_{\mathbf{G}} 0$, if $s \neq j$ or $t \neq k$

b) $S\left(r_{i j k}, r_{l s t}\right) \longrightarrow \mathbf{G} 0$, if $s=j$ and $t=k$.

Lemma 15. a. $S\left(r_{i j k}, r_{l s t u}\right) \longrightarrow_{\mathbf{G}} 0$ for $j \neq l$ and $j \neq s$ or $k \neq u$ and $k \neq t$ 
b. $S\left(r_{i j k}, r_{l s t u}\right) \longrightarrow \mathbf{G} 0$ for $l=j$ and $u=k$

c. $S\left(r_{i j k}, r_{l s t u}\right) \longrightarrow \mathbf{G} 0$ for $j=s$ and $t=k$.

Lemma 16. a. $S\left(r_{i j k}^{\prime}, r_{l s t}^{\prime}\right) \longrightarrow \mathbf{G} 0$ for $l \neq i$ and $s \neq j$ or $k \neq t$

b. $S\left(r_{i j k}^{\prime}, r_{l s t}^{\prime}\right) \longrightarrow \mathbf{G} 0$ for $l=i$, and $t \neq j$ or $k \neq t$

c. $S\left(r_{i j k}^{\prime}, r_{l s t}^{\prime}\right) \longrightarrow_{\mathbf{G}} 0$ for $l \neq i, s=j$ and $t=k$.

Lemma 17. a. $S\left(r_{i j k}^{\prime}, r_{l s t u}\right) \longrightarrow_{\mathbf{G}} 0$ for $j \neq l$ and $j \neq s$ or $k \neq u$ and $k \neq t$

b. $S\left(r_{i j k}^{\prime}, r_{l s t u}\right) \longrightarrow \mathbf{G} 0$ for $l=j$ and $u=k$

c. $S\left(r_{i j k}^{\prime}, r_{l s t u}\right) \longrightarrow \mathbf{G} 0$ for $j=s$ and $t=k$.

Lemma 18. a. $S\left(r_{i j k l}, r_{q s t u}\right) \longrightarrow \mathbf{G} 0$ if, $\operatorname{gcd}\left(w_{j k i l}, w_{s t q u}\right)=1$

b. $S\left(r_{i j k l}, r_{q s t u}\right) \longrightarrow \mathbf{G} 0$, for $q=i$ and $u=l$

c. $S\left(r_{i j k l}, r_{q s t u}\right) \longrightarrow \mathbf{G} 0$, for $s=j$ and $t=l$

d. $S\left(r_{i j k l}, r_{q s t u}\right) \longrightarrow \mathbf{G} 0$, for $q=j$ and $u=k$

e. $S\left(r_{i j k l}, r_{q s t u}\right) \longrightarrow \mathbf{G} 0$, for $s=i$ and $t=l$.

By the above theorem and Theorem 2 the ideal of relations $I$ is generated by $H=$ $G \cap \mathbb{R}[w, z]$. Thus $I=\left\langle r_{i j k}^{\prime}, r_{i j k l}\right\rangle$, where

$$
\begin{aligned}
r_{i j k}^{\prime} & =z_{i} w_{j k}-z_{j} w_{i k}+w_{i j} z_{k}, 1 \leq i<j<k \leq n ; \\
r_{i j k l} & =w_{i l} w_{j k}-w_{i k} w_{j l}+w_{i j} w_{k l}, 1 \leq i<j<k<\leq n .
\end{aligned}
$$

In Step three from the Groebner basis for the ideal of relations $I$, we find a Stanley decomposition for the ring of invariants.

Let $\widetilde{I}=\left\langle\alpha_{i} \beta_{j k}, \beta_{i l} \beta_{j k}\right\rangle$ be the ideal generated by the leading terms of the Groebner basis for I, which is a monomial ideal. It suffices to work with $\mathbb{R}[\alpha, \beta] / \widetilde{I}$ since it is a well known fact that a Stanley decomposition of $\mathbb{R}[\alpha, \beta] / I$ is the same as a Stanley decomposition of $\mathbb{R}[\alpha, \beta] / \widetilde{I}$. See $[13]$ for more details.

As an example we consider a normal form with linear part $N=N_{222}$. Then

$$
x=x_{1} \frac{\partial}{\partial y_{1}}+x_{2} \frac{\partial}{\partial y_{2}}+x_{3} \frac{\partial}{\partial y_{3}}
$$

and the basic invariants are:

$$
\begin{aligned}
\alpha_{i} & =x_{i} ; 1 \leq i \leq 3 \\
\beta_{i j} & =x_{i} y_{j}-y_{i} x_{j} ; 1 \leq i<j \leq 3 .
\end{aligned}
$$

There is only one generator, which therefore forms a Groebner basis, for the ideal of relations among the invariants, $\alpha_{1} \beta_{23}-\alpha_{2} \beta_{13}+\alpha_{3} \beta_{12}=0$ with $\widetilde{I}=\left\langle\alpha_{1} \beta_{23}\right\rangle$. This relation can be used to eliminate the combination $\alpha_{1} \beta_{23}$ (the leading term of the relation) from any polynomial in the invariants. A Stanley decomposition is therefore given by

$$
\frac{\mathbb{R}\left[\beta_{12}, \beta_{13}, \beta_{23}, \alpha_{1}, \alpha_{2}, \alpha_{3}\right]}{\left\langle\alpha_{1} \beta_{23}\right\rangle}=\mathbb{R}\left[\beta_{12}, \beta_{13}, \alpha_{1}, \alpha_{2}, \alpha_{3}\right] \oplus \mathbb{R}\left[\beta_{12}, \beta_{13}, \beta_{23}, \alpha_{2}, \alpha_{3}\right] \beta_{23} .
$$


Writing down Stanley decompositions can sometime be difficult, especially when there is more than one relation among the basic invariants. We describe an easier method of writing down the Stanley decomposition when this be the case.

Definition 5. Let $\mathrm{K}$ be any field and let $P=\left\{x_{1}, x_{2}, \cdots, x_{n}\right\}$ be a partially ordered set (poset). Let $\mathrm{R}=K\left[x_{1}, x_{2}, \cdots, x_{n}\right]$ be a polynomial ring, in which the elements of $\mathrm{P}$ are regarded as the independent indeterminates. Let $I_{P}$ be the ideal of $R$ generated by all products, $x_{i} x_{j}$, such that $x_{i}$ and $x_{j}$ are incomparable as elements of $P$, that is, neither $x_{i} \leq x_{j}$ nor $x_{j} \leq x_{i}$. Setting $R_{P}=R / I_{P}$, we call $R_{P}$ the poset ring corresponding to the poset $P$.

It is not hard to see that our $\operatorname{ring} \mathcal{R}=\mathbb{R}\left[\alpha_{i}, \beta_{i j}\right] / \widetilde{I}, 1 \leq i \leq n$ and $1 \leq i<j \leq n$, is a poset ring corresponding to the poset $P_{n}=\left\{\alpha_{i}, \beta_{i j}\right\}$ with the ordering defined by the relations

$$
\left\{\begin{array}{cl}
\beta_{i j} \leq \beta_{k l}, & \text { if } i \leq k \text { and } j \leq l \\
\alpha_{i} \leq \alpha_{j}, & \text { if } i \leq j \\
\beta_{i j} \leq \alpha_{i}, & \text { for all } i
\end{array}\right.
$$

since the generators of $\widetilde{I}$ are products of incomparable elements of $P_{n}$. For example, $n=4$ the poset $P_{n}$ is diagrammatically

$$
\begin{aligned}
& \alpha_{4} \\
& \uparrow \\
& \alpha_{3} \leftarrow \beta_{34} \\
& \uparrow \uparrow \\
& \alpha_{2} \leftarrow \beta_{24} \leftarrow \beta_{23} \\
& \uparrow \uparrow \uparrow \\
& \alpha_{1} \leftarrow \beta_{14} \leftarrow \beta_{13} \leftarrow \beta_{12},
\end{aligned}
$$

where $s \rightarrow t$ means $s \leq t$. We define paths from $\beta_{12}$ to $\alpha_{4}$ to be any moves in the direction of the arrows, that is to be made up of moves left or moves up. Such paths are called maximal monotone paths.

Every chain takes for example the form

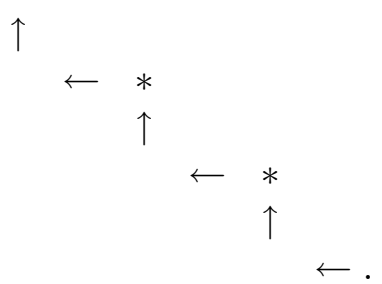

Each of the points marked $*$ is called a corner of a maximal monotone path. A Stanley decomposition of the poset ring $\mathcal{R}$ is then given by:

$$
\mathcal{R}=\bigoplus_{i} \mathbb{R}\left[\text { variables on the } i^{\text {th }} \text { path }\right] \text { (product of corners on the } i^{\text {th }} \text { path). }
$$

The fact that this is indeed a Stanley decomposition follows from Lemma 2.4 in [13]. We revisit the example with linear part $N_{222}$. Recall that, for $\mathrm{n}=3, \widetilde{I}=\left\langle\alpha_{1} \beta_{23}\right\rangle$ and the poset $P_{3}$ of the poset ring $\mathbb{R}[\alpha, \beta] / \widetilde{I}$ is therefore 


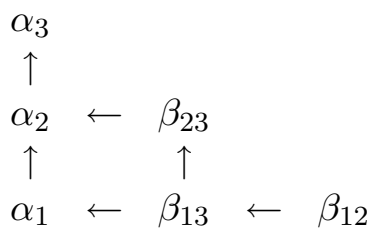

with two maximal monotone paths, $\left(\beta_{12} \rightarrow \beta_{13} \rightarrow \alpha_{1} \rightarrow \alpha_{2} \rightarrow \alpha_{3}\right)$ with no corner and $\left(\beta_{12} \rightarrow \beta_{13} \rightarrow \beta_{23} \rightarrow \alpha_{2} \rightarrow \alpha_{3}\right)$ with $\beta_{23}$ as corner. Hence a Stanley decomposition is easily written down as

$$
\mathcal{R}=\mathbb{R}\left[\beta_{12}, \beta_{13}, \alpha_{1}, \alpha_{2}, \alpha_{3}\right] \oplus \mathbb{R}\left[\beta_{12}, \beta_{13}, \beta_{23}, \alpha_{2}, \alpha_{3}\right] \beta_{23},
$$

which is same as before.

Next we write down a Stanley decomposition for a normal form with linear part $N_{2222}$. We have

$$
X=x_{1} \frac{\partial}{\partial y_{1}}+x_{2} \frac{\partial}{\partial y_{2}}+x_{3} \frac{\partial}{\partial y_{3}}+x_{4} \frac{\partial}{\partial y_{4}}
$$

and the basic invariants are:

$$
\begin{aligned}
\alpha_{i} & =x_{i} ; 1 \leq i \leq 4 \\
\beta_{i j} & =x_{i} y_{j}-y_{i} x_{j} ; 1 \leq i<j \leq 4 .
\end{aligned}
$$

A Groebner basis (generators) for the ideal of relations among the invariants is:

$$
\begin{gathered}
\alpha_{1} \beta_{23}-\alpha_{2} \beta_{13}+\beta_{12} \alpha_{3}=0 \\
\alpha_{1} \beta_{24}-\alpha_{2} \beta_{14}+\beta_{12} \alpha_{4}=0 \\
\alpha_{1} \beta_{34}-\alpha_{2} \beta_{14}+\beta_{13} \alpha_{4}=0 \\
\alpha_{2} \beta_{34}-\alpha_{3} \beta_{24}+\beta_{23} \alpha_{4}=0 \\
\beta_{14} \beta_{23}-\beta_{13} \beta_{24}+\beta_{12} \beta_{34}=0
\end{gathered}
$$

with the monomial ideal $\widetilde{I}=\left\langle\alpha_{1} \beta_{23}, \alpha_{1} \beta_{24}, \alpha_{1} \beta_{34}, \alpha_{2} \beta_{34}, \beta_{14} \beta_{23}\right\rangle$ of the leading terms of $I$. These relations can be used to eliminate any of the leading terms from any polynomial in the invariants, leading to a Stanley decomposition for the $\operatorname{ring} \mathcal{R}=\mathbb{R}[\alpha, \beta] / \widetilde{I}$. The Stanley decomposition is easily obtained from the poset $P_{4}$ given by.

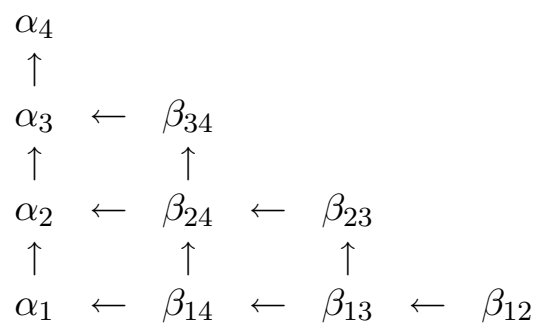

with the following maximal monotone paths:

$\left(\beta_{12} \rightarrow \beta_{13} \rightarrow \beta_{14} \rightarrow \alpha_{1} \rightarrow \alpha_{2} \rightarrow \alpha_{3} \rightarrow \alpha_{4}\right)$, with no corners.

$\left(\beta_{12} \rightarrow \beta_{13} \rightarrow \beta_{23} \rightarrow \beta_{24} \rightarrow \alpha_{2} \rightarrow \alpha_{3} \rightarrow \alpha_{4}\right)$, with $\beta_{23}$ as corner.

$\left(\beta_{12} \rightarrow \beta_{13} \rightarrow \beta_{14} \rightarrow \beta_{24} \rightarrow \alpha_{2} \rightarrow \alpha_{3} \rightarrow \alpha_{4}\right)$, with $\beta_{24}$ as corner. 
$\left(\beta_{12} \rightarrow \beta_{13} \rightarrow \beta_{14} \rightarrow \beta_{24} \rightarrow \beta_{34} \rightarrow \alpha_{3} \rightarrow \alpha_{4}\right)$,with $\beta_{34}$ as corner.

$\left(\beta_{12} \rightarrow \beta_{13} \rightarrow \beta_{23} \rightarrow \beta_{24} \rightarrow \beta_{34} \rightarrow \alpha_{3} \rightarrow \alpha_{4}\right)$, with $\beta_{23}$ and $\beta_{34}$ as corners.

Hence we obtain the following Stanley decomposition for $\mathcal{R}$.

$$
\begin{aligned}
\mathcal{R} & =\mathbb{R}\left[\beta_{12}, \beta_{13}, \beta_{14}, \alpha_{1}, \alpha_{2}, \alpha_{3}, \alpha_{4}\right] \oplus \mathbb{R}\left[\beta_{12}, \beta_{13}, \beta_{23}, \beta_{24}, \alpha_{2}, \alpha_{3}, \alpha_{4}\right] \beta_{23} \\
& \mathbb{R}\left[\beta_{12}, \beta_{13}, \beta_{14}, \beta_{24}, \alpha_{2}, \alpha_{3}, \alpha_{4}\right] \beta_{24} \oplus \mathbb{R}\left[\beta_{12}, \beta_{13}, \beta_{14}, \beta_{24}, \beta_{34}, \alpha_{3}, \alpha_{4}\right] \beta_{34} \\
& \oplus \mathbb{R}\left[\beta_{12}, \beta_{13}, \beta_{23}, \beta_{24}, \beta_{34}, \alpha_{3}, \alpha_{4}\right] \beta_{23} \beta_{34} .
\end{aligned}
$$

Lastly in step four, given a Stanley decomposition for the ring $\mathcal{R}=\mathbb{R}[\alpha, \beta] / \widetilde{I}$, we develop a table function denoted by $T_{2 n}$, where $2 n$ is the dimension of the nilpotent matrix $N_{22 \cdots 2}$. By Lemma 4.7.9 in [10], to prove that $\mathcal{R}=\operatorname{ker} X$, that is to show that we have found all the basic invariants, it suffices to check that $\left.\frac{\partial}{\partial w}\left(w T_{2 n}\right)\right|_{w=1}=\frac{1}{(1-d)^{2 n}}$.

For example from the Stanley decomposition of $N_{2222}$ above another way to say this is that any polynomial $f$ in $\mathcal{R}$ can be written uniquely as

$$
\begin{aligned}
& f=f_{1}\left(\beta_{12}, \beta_{13}, \beta_{14}, \alpha_{1}, \alpha_{2}, \alpha_{3}, \alpha_{4}\right)+f_{2}\left(\beta_{12}, \beta_{13}, \beta_{23}, \beta_{24}, \alpha_{2}, \alpha_{3}, \alpha_{4}\right) \beta_{23} \\
& +f_{3}\left(\beta_{12}, \beta_{13}, \beta_{14}, \beta_{24}, \alpha_{2}, \alpha_{3}, \alpha_{4}\right) \beta_{24}+f_{4}\left(\beta_{12}, \beta_{13}, \beta_{14}, \beta_{24}, \beta_{34}, \alpha_{3}, \alpha_{4}\right) \beta_{34} \\
& +f_{5}\left(\beta_{12}, \beta_{13}, \beta_{23}, \beta_{24}, \beta_{34}, \alpha_{3}, \alpha_{4}\right) \beta_{23} \beta_{34} \text {, }
\end{aligned}
$$

where $f_{1}, f_{2}, f_{3}, f_{4}$ and $f_{5}$ are polynomials in their respective variables. The Stanley decomposition (3.6) can be abbreviated as $f_{1} .1+f_{2} \cdot \beta_{23}+f_{3} \cdot \beta_{24}+f_{4} \cdot \beta_{34}+f_{5} \cdot \beta_{23} \beta_{34}$. The $f s$ are referred to as coefficient functions and $\left\{1, \beta_{23}, \beta_{24}, \beta_{34}, \beta_{23} \beta_{34}\right\}$ as Stanley basis elements. To generate the table function of a Stanley decomposition (for more details see [10] and [9]) we replace each term in (3.6) by a rational function $P / Q$ in $d$ and $w$ (for " $\mathrm{d}=$ degree in $x$ " and "w=weight") constructed as follows. For each basic invariant appearing in a coefficient function, the denominator contains a factor $1-d^{p} w^{q}$, where $p$ and $q$ are the degree and weight of the invariant; the numerator is $d^{p} w^{q}$, where $p$ and $q$ are the degree and weight of the Stanley basis element of that term. When the rational functions $P / Q$ from each term of the Stanley decomposition are summed we obtain the table function $T_{2 n}$ given by $T_{2 n}=\sum_{i} P_{i} / Q_{i}$. Thus for the above example the table function is

$T_{8}=\frac{1}{(1-d w)^{4}\left(1-d^{2}\right)^{3}}+\frac{2 d^{2}}{(1-d w)^{3}\left(1-d^{2}\right)^{4}}+\frac{d^{2}}{(1-d w)^{2}\left(1-d^{2}\right)^{5}}+\frac{d^{2} 4}{(1-d w)^{2}\left(1-d^{2}\right)^{5}}$

and it is easily verified that

$$
\left.\frac{d}{d w}\left(w T_{8}\right)\right|_{w=1}=\frac{1}{(1-d)^{8}}
$$

This shows that all the basic invariants have been found for all $s l(2)$ normal forms with linear part $\mathrm{N}=N_{2222}$. Thus $\mathcal{R}=\operatorname{ker} \mathcal{X}$.

We are now faced with the following question: Can we generalize these results to any given linear part $N_{222 \cdots 2}$, that is, can we claim that we have found all the basic invariants for any normal form with linear part $N_{222 \cdots 2}$ ? To answer this question we proceed as follows: It is clear from the definition of a table function and from the above example that 
for each term of a table function the denominator corresponds to a maximal monotone path and the numerator to the corners associated to that path. Consider a poset ring $\mathcal{R}$ corresponding to the poset $P_{n}=\left\{\alpha_{i}, \beta_{i j}\right\}$ with the ordering defined as before in equation (3.4). The poset $P_{n}$ then looks like

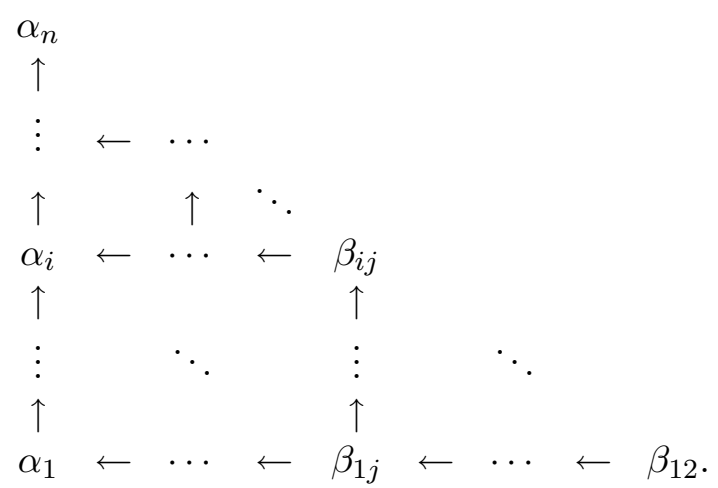

Remark. In each case there is only one maximal monotone path with no corner, namely the outermost path containing all the $\alpha s$.

This brings us to our next question: How many paths starting from $\beta_{12}$, exiting at $\alpha_{i}$ and ending at $\alpha_{n}$, for $i=2,3, \cdots, n-1$, have 1 corner, 2 corners, 3 corners, $\cdots, \mathrm{k}$ corners? This is a problem in combinatorics on the number of lattice paths that never go below the line $y=k x$ for a positive $k$. The result which we repeat here for convenience is known and is given as Theorem 3.4.3 in [7].

Theorem 8. Let $\mu$ be a positive integer and $n \geq \mu m \geq 0$. The number of all lattice paths from $(0,0)$ to $(m, n-1)$ with $k$ up-right corners that never go below the line $y=\mu x$ is

$$
\left(\begin{array}{c}
m-1 \\
k-1
\end{array}\right)\left(\begin{array}{l}
n \\
k
\end{array}\right)-\mu\left(\begin{array}{c}
m \\
k
\end{array}\right)\left(\begin{array}{l}
n-1 \\
k-1
\end{array}\right)
$$

Hence it follows (with $\mu=1$ ) that the number of maximal monotone paths with $k$ corners starting at $\beta_{12}$ and exiting at $\alpha_{i}$, denoted by $C_{n i k}$, is

$$
C_{n i k}=\left(\begin{array}{c}
m-1 \\
k-1
\end{array}\right)\left(\begin{array}{c}
n \\
k
\end{array}\right)-\left(\begin{array}{c}
m \\
k
\end{array}\right)\left(\begin{array}{l}
n-1 \\
k-1
\end{array}\right)=\frac{n-i}{k}\left(\begin{array}{c}
n-2 \\
k-1
\end{array}\right)\left(\begin{array}{c}
i-2 \\
k-1
\end{array}\right) .
$$

The following theorem shows that for $n \geq 2$ all the basic invariants for coupled TakensBogdanov systems have been found.

Theorem 9. (Ring of invariants) Let $\mathcal{R}=\mathbb{R}\left[\alpha_{i}, \beta_{i j}\right] / \widetilde{I}$, where $\widetilde{I}=\left\langle\alpha_{i} \beta_{j k}, \beta_{i l} \beta_{j k}\right\rangle$ for $1 \leq i \leq n, 1 \leq i<j \leq n$ and for $i<j<k, i<j<k<l$. Then $\mathcal{R}=\operatorname{ker} \mathcal{X}$, the ring of invariants.

Proof. The table function for $\mathcal{R}$ is

$$
T_{2 n}=\frac{1}{\left(1-d^{2}\right)^{n-1}(1-d w)^{n}}+\sum_{k=1}^{n-1} \sum_{i=k+1}^{n-1} \frac{C_{n i k} d^{2 k}}{\left(1-d^{2}\right)^{n+i-2}(1-d w)^{n-i+1}},
$$


where $C_{n i k}$ is as given in (3.7).

We need to verify that

$$
\left.\frac{\partial}{\partial w}\left(w T_{2 n}\right)\right|_{w=1}=\frac{1}{(1-d)^{2 n}}
$$

Calculating the lhs of (3.9) we get

$$
\begin{array}{r}
\frac{1}{(1+d)^{2 n-3}(1-d)^{2 n}}\left[(1+(n-1) d)(1+d)^{n-2}\right. \\
\left.+\sum_{k=1}^{n-1} \sum_{i=k+1}^{n-1} C_{n i k}(1+(n-i) d)(1+d)^{n-i-2} d^{2 k}\right]=\frac{1}{(1-d)^{2 n}} .
\end{array}
$$

After simplifying (3.10), it is enough to prove the identity,

$$
(1+(n-1) d)(1+d)^{n-2}+\sum_{k=1}^{n-1} \sum_{i=k+1}^{n-1} C_{n i k}(1+(n-i) d)(1+d)^{n-i-1} d^{2 k}=(1+d)^{2 n-3}
$$

or equivalently (after rearranging) the identity,

$$
\sum_{k=1}^{n-1} \sum_{i=k+1}^{n-1} C_{n i k}(1+(n-i) d)(1+d)^{n-i-1} d^{2 k}=(1+d)^{2 n-3}-(1+(n-1) d)(1+d)^{n-2}
$$

Define $\mathrm{F}(\mathrm{n}, \mathrm{k})=\sum_{r=k+1}^{n-1} C_{n i k}(1+(n-i) d)(1+d)^{n-i-1} d^{2 k}$ and let $f(n)=\sum_{k=1}^{n-1} F(n, k)$. Since $f(n)$ is hypergeometric, see [12], and by Zeilberger's algorithm found in [11], its recurrence relation is

$$
\left\{-d(1+d)^{2} f(n)+d f(n+1)=d^{3}(1+d)^{n-1}(n-1)\right\}
$$

Rearranging (3.13), we find that the lhs of (3.12) satisfies the recurrence,

$$
f(n+1)-(1+d)^{2} f(n)=(n-1) d^{2}(1+d)^{n-1} .
$$

Next we show that the rhs of (3.12) satisfies the same recurrence relation. Since $r h s=$ $f(n)=(1+d)^{2 n-3}-(1+(n-1) d)(1+d)^{n-2}$,

$$
\begin{aligned}
& (1+d)^{2} f(n)=(1+d)^{2 n-1}-(1+(n-1) d)(1+d)^{n} \\
& f(n+1)=(1+d)^{2 n-1}-(1+n d)(1+d)^{n-1} .
\end{aligned}
$$

Subtracting (3.15) from (3.16) and simplifying we get

$$
f(n+1)-(1+d)^{2} f(n)=(n-1) d^{2}(1+d)^{n-1}=\text { lhs }
$$

Hence we have shown that both sides satisfy the same recurrence. Lastly, we check that both sides satisfy the initial condition $n=2$ since we have that $n \geq 2$. Let $L(n)=l h s$ and $R(n)=r h s$ of (3.12). Clearly $\mathrm{L}(2)=0$ and $\mathrm{R}(2)=0$. This establishes the identity and hence proves the theorem. 


\section{Stanley Decomposition for ker $\mathcal{D}^{r}$ and for Normal Form Module ker X}

In this section we apply Murdock's algorithm developed in [10], section 4.7, to obtain the Stanley decomposition of the module ker $X^{r}$. Then using Theorem 3 we obtain a Stanley decomposition of the normal form module of equivariants ker $\mathrm{X}$ from the Stanley decomposition of ker $X^{r}$.

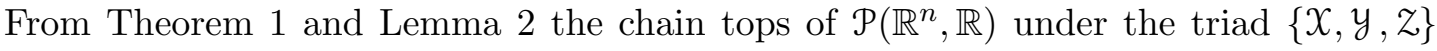
can be taken to be the standard monomials in the basic invariants, say $I_{1}, I_{2}, \cdots, I_{s}$, with respect to the Stanley decomposition of ker $X$. The chains under these chain tops are the lower Jordan chains of $y$ and can be obtained by repeated application of $y$. These chains are also modified upper Jordan chains for $\mathcal{X}$ (regarded as a mapping upwards). Clearly a vector space basis for ker $X^{r}$ is obtained by computing the $y$ iterates to depth $r$.

Remark. By modified Jordan chains for $\mathcal{X}$ we mean that $\mathcal{X}$ operating on a given vector in the chain gives a nonzero constant times the vector above it, or zero if the given vector is at the top of a chain.

We now describe how to obtain a Stanley decomposition of ker $X^{r}$ from any Stanley decomposition of ker $X$.

\section{Algorithm to find ker $X^{r}$}

The following four steps apply:

1. Order the basic invariants putting the longest (with largest $r$ ) basic invariant last (to the far right).

2. Determine the finite set of suffixes that can occur to depth $r$. All standard monomials can be classified by their endings. These endings determine the suffixes that can occur when the $y_{s}$ are applied repeatedly from the last according to Lemma 3.

3. Determine the prefix ring (coefficient ring) for each suffix. The prefix ring for a given suffix is the set of admissible prefixes (standard monomials not containing the endings) that can appear with that suffix.

4. Write down a Stanley decomposition for ker $X^{r}$ as given by Theorem 4 , that is

$$
\operatorname{ker} X^{r}=S D(\operatorname{ker} X) \oplus\left(\bigoplus_{S} P(S) S\right)
$$

where $S D(\operatorname{ker} \mathcal{X})$ is the Stanley decomposition of ker $\mathcal{X}$ and $P(S)$ is the Stanley decomposition of the prefix ring corresponding to suffix $S$.

By example $N_{22 \cdots 2}$ we mean a coupled Takens-Bogdanov system with linear part $N_{22 \cdots 2}$.

Example $N_{222}$. The basic invariants for $N_{222}$ are:

$$
\begin{aligned}
\alpha_{i} & =x_{i} ; 1 \leq i \leq 3 \\
\beta_{i j} & =x_{i} y_{j}-y_{i} x_{j} ; 1 \leq i<j \leq 3 .
\end{aligned}
$$


These are related by the single relation $\alpha_{1} \beta_{23}-\alpha_{2} \beta_{13}+\alpha_{3} \beta_{12}=0$, with $\alpha_{1} \beta_{23}$ as the leading monomial. To compute ker $X^{2}$ it is necessary to apply $y$ to depth 2 . Order the basic invariants by $\beta_{23}<\beta_{13}<\beta_{12}<\alpha_{3}<\alpha_{2}<\alpha_{1}$, with length 1, 1, 1, 2, 2, and 2 respectively. The standard monomials cannot contain both $\alpha_{1}$ and $\beta_{23}$ and can be classified into those ending in either $\alpha_{1}, \alpha_{2}$ or $\alpha_{3}$ and those ending in either of the trivial basic invariants $\beta_{12}, \beta_{13}$ or $\beta_{23}$. For those ending in $\alpha_{1}$ the suffix is $y \alpha_{1}$ and the prefix ring is $\mathbb{R}\left[\alpha_{1}, \alpha_{2}, \alpha_{3}, \beta_{12}, \beta_{13}\right]$, for those ending in $\alpha_{2}$ the suffix is $y \alpha_{2}$ and the prefix ring is $\mathbb{R}\left[\alpha_{2}, \alpha_{3}, \beta_{12}, \beta_{13}\right] \oplus \mathbb{R}\left[\alpha_{2}, \alpha_{3}, \beta_{12}, \beta_{13}, \beta_{23}\right] \beta_{23}$ and for those ending in $\alpha_{3}$ the suffix is $y \alpha_{3}$ and the prefix ring is $\mathbb{R}\left[\alpha_{3}, \beta_{12}, \beta_{13}\right] \oplus \mathbb{R}\left[\alpha_{3}, \beta_{12}, \beta_{13}, \beta_{23}\right] \beta_{23}$. Since the standard monomials ending in either of the trivial basic invariants have no suffixes, the suffix set is $\left\{y \alpha_{1}, y \alpha_{2}, y \alpha_{3}\right\}$. The Stanley decomposition for the module ker $X^{2}$ according to Theorem 4 is therefore

$$
\begin{aligned}
\operatorname{ker} \mathcal{X}^{2} & =\mathbb{R}\left[\alpha_{1}, \alpha_{2}, \alpha_{3}, \beta_{12}, \beta_{13}\right] \oplus \mathbb{R}\left[\alpha_{2}, \alpha_{3}, \beta_{12}, \beta_{13}, \beta_{23}\right] \beta_{23} \\
& \oplus \mathbb{R}\left[\alpha_{1}, \alpha_{2}, \alpha_{3}, \beta_{12}, \beta_{13}\right] \text { y } \alpha_{1} \\
& \oplus\left(\mathbb{R}\left[\alpha_{2}, \alpha_{3}, \beta_{12}, \beta_{13}\right] \oplus \mathbb{R}\left[\alpha_{2}, \alpha_{3}, \beta_{12}, \beta_{13}, \beta_{23}\right] \beta_{23}\right) \text { y } \alpha_{2} \\
& \oplus\left(\mathbb{R}\left[\alpha_{3}, \beta_{12}, \beta_{13}\right] \oplus \mathbb{R}\left[\alpha_{3}, \beta_{12}, \beta_{13}, \beta_{23}\right] \beta_{23}\right) \text { y } \alpha_{3} .
\end{aligned}
$$

After obtaining the Stanley decomposition for ker $X^{2}$, the $s l(2)$ normal form ker $\mathrm{X}$, that is, the module of equivariants follows easily from Theorem 3 . Recall that

ker $\mathrm{X} \cong \operatorname{ker} X^{r_{1}} \oplus \operatorname{ker} X^{r_{2}} \oplus \cdots \oplus \operatorname{ker} X^{r_{k}}$. Hence For $N_{222}$ we have

ker $\mathrm{X} \cong \operatorname{ker} X^{2} \oplus \operatorname{ker} X^{2} \oplus \operatorname{ker} X^{2}$. Explicitly by Lemma 1 the Stanley decomposition for the $\operatorname{sl}(2)$ normal form is therefore

$$
\begin{aligned}
\operatorname{ker} X & =\left(\mathbb{R}\left[\alpha_{1}, \alpha_{2}, \alpha_{3}, \beta_{12}, \beta_{13}\right] \oplus \mathbb{R}\left[\alpha_{2}, \alpha_{3}, \beta_{12}, \beta_{13}, \beta_{23}\right] \beta_{23}\right) v_{\{1,1\}} \\
& \oplus \mathbb{R}\left[\alpha_{1}, \alpha_{2}, \alpha_{3}, \beta_{12}, \beta_{13}\right] v_{\left\{1, y \alpha_{1}\right\}} \\
& \oplus\left(\mathbb{R}\left[\alpha_{2}, \alpha_{3}, \beta_{12}, \beta_{13}\right] \oplus \mathbb{R}\left[\alpha_{2}, \alpha_{3}, \beta_{12}, \beta_{13}, \beta_{23}\right] \beta_{23}\right) v_{\left\{1, y \alpha_{2}\right\}} \\
& \oplus\left(\mathbb{R}\left[\alpha_{3}, \beta_{12}, \beta_{13}\right] \oplus \mathbb{R}\left[\alpha_{3}, \beta_{12}, \beta_{13}, \beta_{23}\right] \beta_{23}\right) v_{\left\{1, y \alpha_{3}\right\}} \\
& \oplus\left(\mathbb{R}\left[\alpha_{1}, \alpha_{2}, \alpha_{3}, \beta_{12}, \beta_{13}\right] \oplus \mathbb{R}\left[\alpha_{2}, \alpha_{3}, \beta_{12}, \beta_{13}, \beta_{23}\right] \beta_{23}\right) v_{\{2,2\}} \\
& \oplus \mathbb{R}\left[\alpha_{1}, \alpha_{2}, \alpha_{3}, \beta_{12}, \beta_{13}\right] v_{\left\{2, y \alpha_{1}\right\}} \\
& \oplus\left(\mathbb{R}\left[\alpha_{2}, \alpha_{3}, \beta_{12}, \beta_{13}\right] \oplus \mathbb{R}\left[\alpha_{2}, \alpha_{3}, \beta_{12}, \beta_{13}, \beta_{23}\right] \beta_{23}\right) v_{\left\{2, y \alpha_{2}\right\}} \\
& \oplus\left(\mathbb{R}\left[\alpha_{3}, \beta_{12}, \beta_{13}\right] \oplus \mathbb{R}\left[\alpha_{3}, \beta_{12}, \beta_{13}, \beta_{23}\right] \beta_{23}\right) v_{\left\{2, y \alpha_{3}\right\}} \\
& \oplus\left(\mathbb{R}\left[\alpha_{1}, \alpha_{2}, \alpha_{3}, \beta_{12}, \beta_{13}\right] \oplus \mathbb{R}\left[\alpha_{2}, \alpha_{3}, \beta_{12}, \beta_{13}, \beta_{23}\right] \beta_{23}\right) v_{\{3,3\}} \\
& \oplus \mathbb{R}\left[\alpha_{1}, \alpha_{2}, \alpha_{3}, \beta_{12}, \beta_{13}\right] v_{\left\{3, y \alpha_{1}\right\}} \\
& \oplus\left(\mathbb{R}\left[\alpha_{2}, \alpha_{3}, \beta_{12}, \beta_{13}\right] \oplus \mathbb{R}\left[\alpha_{2}, \alpha_{3}, \beta_{12}, \beta_{13}, \beta_{23}\right] \beta_{23}\right) v_{\left\{3, y \alpha_{2}\right\}} \\
& \left.\oplus\left(\mathbb{R}\left[\alpha_{3}, \beta_{12}, \beta_{13}\right] \oplus \mathbb{R}\left[\alpha_{3}, \beta_{12}, \beta_{13}, \beta_{23}\right] \beta_{23}\right) v_{\{3, y} \alpha_{3}\right\}
\end{aligned}
$$

Here

$$
v_{\{1,1\}}=\left[\begin{array}{c}
0 \\
1 \\
0 \\
0 \\
0 \\
0
\end{array}\right], \quad v_{\left\{1, y \alpha_{1}\right\}}=\left[\begin{array}{c}
x y \alpha_{1} \\
y \alpha_{1} \\
0 \\
0 \\
0 \\
0
\end{array}\right], \quad v_{\left\{1, y \alpha_{2}\right\}}=\left[\begin{array}{c}
x y \alpha_{2} \\
y \alpha_{2} \\
0 \\
0 \\
0 \\
0
\end{array}\right], \quad v_{\left\{1, y \alpha_{3}\right\}}=\left[\begin{array}{c}
x y \alpha_{3} \\
y \alpha_{3} \\
0 \\
0 \\
0 \\
0
\end{array}\right]
$$


$v_{\{2,2\}}=\left[\begin{array}{l}0 \\ 0 \\ 0 \\ 1 \\ 0 \\ 0\end{array}\right], \quad v_{\left\{2, y \alpha_{1}\right\}}=\left[\begin{array}{c}0 \\ 0 \\ x y \alpha_{1} \\ y \alpha_{1} \\ 0 \\ 0\end{array}\right], \quad v_{\left\{2, y \alpha_{2}\right\}}=\left[\begin{array}{c}0 \\ 0 \\ x y \alpha_{2} \\ y \alpha_{2} \\ 0 \\ 0\end{array}\right], \quad v_{\left\{2, y \alpha_{3}\right\}}=\left[\begin{array}{c}0 \\ 0 \\ x y \alpha_{3} \\ y \alpha_{3} \\ 0 \\ 0\end{array}\right]$,
$v_{\{3,3\}}=\left[\begin{array}{l}0 \\ 0 \\ 0 \\ 0 \\ 1\end{array}\right], \quad v_{\left\{3, y \alpha_{1}\right\}}=\left[\begin{array}{c}0 \\ 0 \\ 0 \\ 0 \\ x y \alpha_{1} \\ y \alpha_{1}\end{array}\right], \quad v_{\left\{3, y \alpha_{2}\right\}}=\left[\begin{array}{c}0 \\ 0 \\ 0 \\ 0 \\ x y \alpha_{2} \\ y \alpha_{2}\end{array}\right], \quad v_{\left\{3, y \alpha_{3}\right\}}=\left[\begin{array}{c}0 \\ 0 \\ 0 \\ 0 \\ x y \alpha_{3} \\ y \alpha_{3}\end{array}\right]$.

Example $N_{2222}$. The basic invariants for $N_{2222}$ are:

$$
\begin{aligned}
\alpha_{i} & =x_{i} ; 1 \leq i \leq 4 ; \\
\beta_{i j} & =x_{i} y_{j}-y_{i} x_{j} ; 1 \leq i<j \leq 4 .
\end{aligned}
$$

A Groebner basis for the ideal of relations is:

$$
\begin{gathered}
\alpha_{1} \beta_{23}-\alpha_{2} \beta_{13}+\beta_{12} \alpha_{3}=0 \\
\alpha_{1} \beta_{24}-\alpha_{2} \beta_{14}+\beta_{12} \alpha_{4}=0 \\
\alpha_{1} \beta_{34}-\alpha_{2} \beta_{14}+\beta_{13} \alpha_{4}=0 \\
\alpha_{2} \beta_{34}-\alpha_{3} \beta_{24}+\beta_{23} \alpha_{4}=0 \\
\beta_{14} \beta_{23}-\beta_{13} \beta_{24}+\beta_{12} \beta_{34}=0
\end{gathered}
$$

To compute ker $X^{2}$ it is necessary to apply $y$ to depth 2 . Order the basic invariants by $\beta_{j k}<\alpha_{i}$ for all $i$ and $\alpha_{j}<\alpha_{i}$ for $i<j$. The ordering on the $\beta_{i j}$ is not necessary in our case since each $\beta_{i j}$ is of length 1 and contributes no suffixes. The standard monomials can be classified into those ending in one of the $\alpha_{i}$ s and those ending in either of the trivial basic invariants. For those ending in $\alpha_{i}$ the suffix is $y \alpha_{i}$ (since each $\alpha_{i}$ is of length 2) and those ending in either of the trivial basic invariants have no suffixes. So the suffix set is $\left\{y \alpha_{i}\right.$, for $\left.1 \leq i \leq 4\right\}$. The Stanley decomposition for the module ker $X^{2}$ according to Theorem 4 is therefore

$$
\begin{aligned}
\operatorname{ker} X^{2} & =\mathbb{R}\left[\alpha_{1}, \alpha_{2}, \alpha_{3}, \alpha_{4}, \beta_{12}, \beta_{13}, \beta_{14}\right] \oplus \mathbb{R}\left[\alpha_{2}, \alpha_{3}, \alpha_{4}, \beta_{12}, \beta_{13}, \beta_{23}, \beta_{24}\right] \beta_{23} \\
& \oplus \mathbb{R}\left[\alpha_{2}, \alpha_{3}, \alpha_{4}, \beta_{12}, \beta_{13}, \beta_{14}, \beta_{24}\right] \beta_{24} \oplus \mathbb{R}\left[\alpha_{3}, \alpha_{4}, \beta_{12}, \beta_{13}, \beta_{14}, \beta_{24}, \beta_{34}\right] \beta_{34} \\
& \oplus \mathbb{R}\left[\alpha_{3}, \alpha_{4}, \beta_{12}, \beta_{13}, \beta_{23}, \beta_{24}, \beta_{34}\right] \beta_{23} \beta_{34} \\
& \oplus \mathbb{R}\left[\alpha_{1}, \alpha_{2}, \alpha_{3}, \alpha_{4}, \beta_{12}, \beta_{13}, \beta_{14}\right] y \alpha_{1} \oplus\left(R\left[\alpha_{2}, \alpha_{3}, \alpha_{4}, \beta_{12}, \beta_{13}, \beta_{14}\right]\right. \\
& \left.\oplus \mathbb{R}\left[\alpha_{2}, \alpha_{3}, \alpha_{4}, \beta_{12}, \beta_{13}, \beta_{23}, \beta_{24}\right] \beta_{23} \oplus \mathbb{R}\left[\alpha_{2}, \alpha_{3}, \alpha_{4}, \beta_{12}, \beta_{13}, \beta_{14}, \beta_{24}\right] \beta_{24}\right) y \alpha_{2} \\
& \oplus \mathbb{R}\left[\alpha_{3}, \alpha_{4}, \beta_{12}, \beta_{13}, \beta_{14}\right] \oplus \mathbb{R}\left[\alpha_{3}, \alpha_{4}, \beta_{12}, \beta_{13}, \beta_{23}, \beta_{24}\right] \beta_{23} \\
& \oplus \mathbb{R}\left[\alpha_{2}, \alpha_{3}, \alpha_{4}, \beta_{12}, \beta_{13}, \beta_{14}, \beta_{24}\right] \beta_{24} \oplus \mathbb{R}\left[\alpha_{3}, \alpha_{4}, \beta_{12}, \beta_{13}, \beta_{14}, \beta_{24}, \beta_{34}\right] \beta_{34} \\
& \left.\oplus \mathbb{R}\left[\alpha_{3}, \alpha_{4}, \beta_{12}, \beta_{13}, \beta_{23}, \beta_{24}, \beta_{34}\right] \beta_{23} \beta_{34}\right) y \alpha_{3} \\
& \oplus \mathbb{R}\left[\alpha_{4}, \beta_{12}, \beta_{13}, \beta_{14}\right] \oplus \mathbb{R}\left[\alpha_{4}, \beta_{12}, \beta_{13}, \beta_{23}, \beta_{24}\right] \beta_{23} \oplus \mathbb{R}\left[\alpha_{4}, \beta_{12}, \beta_{13}, \beta_{14}, \beta_{24}\right] \beta_{24} \\
& \left.\oplus \mathbb{R}\left[\alpha_{4}, \beta_{12}, \beta_{13}, \beta_{14}, \beta_{24}, \beta_{34}\right] \beta_{34} \oplus \mathbb{R}\left[\alpha_{4}, \beta_{12}, \beta_{13}, \beta_{23}, \beta_{24}, \beta_{34}\right] \beta_{23} \beta_{34}\right) y \alpha_{4}
\end{aligned}
$$


After obtaining the Stanley decomposition for ker $X^{2}$, the Stanley decomposition for the $s l(2)$ normal form ker $\mathrm{X}$, the module of equivariants follows easily from Theorem 3 . For $N_{2222}$ we have $\operatorname{ker} X \cong \operatorname{ker} X^{2} \oplus \operatorname{ker} X^{2} \oplus \operatorname{ker} X^{2} \oplus \operatorname{ker} X^{2}$. The Stanley decomposition for the $s l(2)$ normal form is obtained by placing equation (4.3) into the second, fourth, sixth and eighth positions in the vector fields and then applying the reconstruction map (2.16) $\left.\left(0, y \alpha_{1}, 0, y \alpha_{2}, 0, y \alpha_{3}, 0, y \alpha_{4}\right) \rightarrow\left(X\left(y \alpha_{1}\right), y \alpha_{1}, X\left(y \alpha_{2}\right), y \alpha_{2}, X\left(y \alpha_{3}\right), y \alpha_{3}, X\left(y \alpha_{4}\right), y \alpha_{14}\right)\right)$. It is clear from the above examples that we can now find the Stanley decomposition for the $\operatorname{sl}(2)$ module of equivariants for any coupled Takens-Bogdanov system. We generalize the above results to any $N_{222 \cdots 2}$ with an arbitrary number of $2 \times 2$ blocks.

Example $N_{222 \cdots 2}$. In this case the basic invariants are

$$
\begin{gathered}
\alpha_{i}=x_{i} \text { for } 1 \leq I \leq n \text { and } \\
\beta_{i j}=x_{i} y_{j}-x_{j} y_{i} \text { for } 1 \leq i<j \leq n .
\end{gathered}
$$

By Theorem 7 the Groebner basis for the ideal of relation is therefore

$$
\mathrm{H}=\left\langle r_{i j k}^{\prime}, r_{i j k l}\right\rangle
$$

where

$$
\begin{aligned}
r_{i j k}^{\prime} & =\alpha_{i} \beta_{j k}-\beta_{j} w_{i k}+\beta_{i j} z_{k}, 1 \leq i<j<k \leq n ; \\
r_{i j k l} & =\beta_{i l} w_{j k}-\beta_{i k} w_{j l}+\beta_{i j} w_{k l}, 1 \leq i<j<k<\leq n,
\end{aligned}
$$

with the monomial ideal $\widetilde{I}=\left\langle\alpha_{i} \beta_{j k}, \beta_{i l} \beta_{j k}\right\rangle$. To compute ker $X^{2}$ it is necessary to apply $y$ to depth 2. Order the basic invariants by $\beta_{j k}<\alpha_{i}$ for all $i$ and $\alpha_{j}<\alpha_{i}$ for $i<j$. Each $\beta_{i j}$ is of length 1 and each $\alpha_{i}$ is of length 2 . The standard monomials can be classified into those ending in one of the $\alpha_{i} \mathrm{~s}$ and those ending in one of the trivial basic invariants. For those ending in $\alpha_{i}$ the suffix is $y \alpha_{i}$ and those ending in either of the trivial basic invariants have no suffixes. Therefore the suffix set is $\left\{y \alpha_{i}\right.$, for $\left.1 \leq i \leq n\right\}$ and by Theorem 4 a Stanley decomposition for the module ker $X^{2}$ is

$$
\text { ker } x^{2}=\operatorname{ker} x \oplus\left(\bigoplus_{i}^{n}\left(\operatorname{ker} x / J_{i}\right) y \alpha_{i}\right) \text {. }
$$

Now the Stanley decomposition for the module of equivariants for $N_{222 \cdots 2}$ is obtained by placing equation (4.4) into the second, fourth, $\cdots$ and $(2 \mathrm{n})$ th positions in the vector fields and then applying the reconstruction map (2.16).

\section{References}

[1] Adams, William W. and Loustaunau, P. (1994). An Introduction to Groebner Bases. American Mathematical Society, Providence.

[2] Cox, David., Little, John and O'Shea, Donal. (1997). Ideals, Varieties and Algorithm. Springer, New York. 
[3] Cushman, R. and Sanders, J. A. (1990). A Survey of Invariant Theory Applied to Normal Forms of Vectorfields with Nilpotent Linear Part, "Invariant Theory and Tableaux" (D. Stanton, Ed.) Springer-Verlag, New York.

[4] Cushman, R. Sanders, J. A. and White, N. (1988). Normal Form for the $(2 ; n)$ Nilpotent Vectorfield, Using Invariant Theory. Physica D 30, 399-412.

[5] Fulton, W. and Harries, Joe.(1991). Representation Theory: A First Course. Springer, New York.

[6] Gatermann, Karen (2000). Computer Algebra Methods for Equivariant Dynamical Systems. Lecture Notes in Mathematics, volume 1728, Springer-Verlag, New York.

[7] Krattenthaler, C. (1997). The Enumeration of Lattice Paths with Respect to Thier Number of Turns, in "Advances in Combinatorial Methods and Applications to Probability and Statistics", N. Balakrishnan, Ed., Berkhäuser, Boston, p. 29-58.

[8] Malonza, David M. (2003). Normal Forms for Coupled Takens-Bogdanov Systems. Ph.D Thesis, Iowa State University, Ames, Iowa, USA

[9] Murdock, James (2002). On the Structure of Nilpotent Normal Form Modules. Journal of Differential Equations 180, 198-237.

[10] Murdock, James (2003). Normal Forms and Unfoldings for Local Dynamical Systems. Springer-Verlag, New York.

[11] Paule, P. and Schorn, M. (1995). A Mathematica Version of Zeilberger's Algorithm for Proving Binomial Coefficient Identities. Journal of Symbolic Computation, 20, 673-698.

[12] Petkov $\breve{s}$, M., Wilf, H. and Zeilberger, D. (1996). A=B. A K Peters Ltd., Wellesley, Massachusetts.

[13] Sturmfels, B. and White, N.(1991). Computing Combinatorial Decompositions of Rings. Combinatorica 11, 275-293.

[14] Sri Namachchivaya, N., Doyle, Monica M., Langford, Willam F. and Evans, Nolan W. (1994). Normal Form for Generalized Hopf Bifurcation with Nonsemisimple 1:1 Resonance. Z. Angew. Math. Phys. 45, no. 2, 312-335. 